\title{
Tesis de Maestría
}

\section{Maestría en Docencia de la Química}

\section{Departamento de Química}

\section{Estado del arte de las investigaciones sobre didáctica de las ciencias experimentales financiadas por Colciencias, 1999-2003}

Giovanna del Pilar Fuentes Medina

Descripción: La investigación tuvo como fin caracterizar los estudios que Colciencias aprobó durante el periodo comprendido entre 1999 y el 2003 dentro de la nueva didáctica de las ciencias experimentales como una disciplina teóricamente fundamentada a través de la determinación del apoyo bibliográfico dado por revistas especializadas, la conformación de una comunidad académica, la participación en seminarios, congresos y simposios. De esta manera, fue posible establecer el estado actual de dicha disciplina por los aportes generados.

Contenido: La nueva didáctica de las ciencias experimentales ha tenido un desarrollo histórico para finalmente establecerse como una disciplina teóricamente fundamentada. El estatuto establecido reconoce que los conceptos científicos son construidos dentro de una comunidad de especialistas que publican sus acuerdos en revistas especializadas y asisten a congresos, seminarios y simposios, tanto en el ámbito nacional como internacional. Al respecto, se encontró que las investigaciones que Colciencias financió reconocen implícitamente la nueva didáctica de las ciencias, ya que se respaldan en artículos publicados en las revistas especializadas, siendo claro que el número de estas referencias es mayor con relación al número de libros consultados especialmente durante el ultimo año; también porque se conforman grupos de investigación con campos de conocimiento definidos, que coinciden con los intereses de sus pares internacionales.

Se elaboraron Resúmenes Analíticos Educativos, RAE para cada una de las investigaciones consultadas. A partir de esta organización, fue posible sistematizar la información, según 3 descriptores: el primero, apoyo bibliográfico en revistas especializadas; segundo, determinación del estado conceptual y metodológico de las investigaciones y finalmente, el aporte a la didáctica de las ciencias.

Conclusiones: Las investigaciones que Colciencias financia, implícitamente se inscriben en la didáctica de las ciencias como disciplina teóricamente fundamentada, a pesar que 2 de los 8 trabajos consultados reconozcan abiertamente a la disciplina como tal. En cuanto al apoyo en revistas especializadas, la didáctica plantea la pertinencia de estas referencias, que fueron publicadas cinco años atrás a la fecha de la presentación de los resultados; sin embargo, la tendencia es a referirse a artículos con 15 años de publicación. De la misma manera, se hacen aportes a líneas de investigación, como resolución de problemas, concepciones alternativas, concepciones epistemológicas de profesores y estudiantes, y formación inicial y continua de profesores. 


\section{El modelo atómico de Ernest Rutherford. Del saber sabio al saber escolar}

Luigi Hawer Cuéllar Fernández

Descripción: En esta investigación se presentan aspectos relacionados con la transposición didáctica del modelo atómico de Ernest Rutherford y el análisis de la confiabilidad de los libros de texto más utilizados por profesores de química en ejercicio en algunos colegios de Bogotá, en educación Media, lo mismo que de las Facultades de Formación de Licenciados en Química de las Universidades Pedagógica Nacional y Distrital Francisco José de Caldas, de Bogotá, desde un análisis histórico-epistemológico. A partir del estudio de los documentos originales se establecieron diez criterios de análisis, clasificados en cinco categorías, que fueron validados por especialistas y fueron la base para determinar la relación existente entre lo que propuso Ernest Rutherford en relación con la estructura del átomo y lo que se socializa en el sistema educativo colombiano, a través de los libros de texto.

Contenidos: Esta investigación está constituida por unos antecedentes relacionados con trabajos sobre transposición didáctica, según una nueva interpretación, desde las ciencias de la naturaleza, de la propuesta de Ives Chevallard (1985) y por las fundamentaciones epistemológicas, didácticas y pedagógicas en las que se apoya. Se desarrollan importantes formulaciones que tienen que ver con la enseñabilidad de las ciencias, la categoría epistemológica de modelo y la importancia de la historia y la filosofía de la ciencia.
Metodología: A partir del estudio histórico-epistemológico de los documentos originales en los que Ernest Rutherford presentó su propuesta sobre la estructura del átomo, se determinaron diez criterios, clasificados en cinco categorías de análisis, a partir de los que pudo establecerse la imagen de ciencia que se socializa en el sistema educativo colombiano. Para tal efecto, se seleccionaron quince libros de texto, cinco de Educación Media y diez de Educación Superior (de los más utilizados en las Universidades Pedagógica Nacional y Distrital Francisco José de Caldas, de Bogotá). Para cada uno de esos libros de texto se elaboró un análisis riguroso sobre la forma como es presentada la formulación de Ernest Rutherford, teniendo como referente lo postulado por él, de conformidad con los criterios establecidos y validados por especialistas en historia y epistemología de las ciencias.

Conclusiones: A partir del estudio de la transposición didáctica, se ha propuesto en esta investigación que ésta se encuentra directamente relacionada con la problemática de la enseñabilidad de las ciencias y que involucra por lo menos tres componentes fundamentales: las intencionalidades curriculares, los modelos didácticos y la confiabilidad o el análisis de los libros de texto.

En lo que respecta a la utilización de los libros de texto en la enseñanza media pudo determinarse que los más utilizados por los profesores de química de los colegios seleccionados corresponden a aquellos en los que los contenidos o áreas temáticas están "ajustados" a los estándares curri- 
culares y a las competencias establecidas por el Ministerio de Educación Nacional.

Así, pues, se establece una visión de ciencia como una actividad simplificada, individual, absolutista y reduccionista, alejada de una realidad que puede ser considerada a partir del acercamiento al reconocimiento del contexto histórico en el que cada uno de estos científicos presentó sus respectivas propuestas. Una mayor rigurosidad en el desarrollo de la temática del modelo atómico propuesto por E. Rutherford en los libros de texto utilizados en educación superior. No obstante, cabe resaltar que sólo uno de los diez libros de texto reportados como los más utilizados es un libro especializado en el tema, y que ninguno de los profesores entrevistados hizo alusión a la utilización de los documentos originales en los que Rutherford presentó sus investigaciones.

En términos generales, pudo corroborarse positivamente el hecho de que en el sistema educativo colombiano la socialización del modelo atómico propuesto por E. Rutherford no obedece a una mirada histórico-epistemológica, identificable a partir del reconocimiento de los documentos originales en los que presentó sus propuestas. Habría que mencionar que de los libros de texto analizados, aquellos que se alejan de una perspectiva histórico-epistemológica introducen errores conceptuales, lo que no los hace confiables $y$, en definitiva, se alejan de lo validado y aceptado por la comunidad de especialistas.

\section{Los conceptos de calor y temperatura desde la enseñanza de las competencias cognoscitivas y los estándares de competencias}

Carmen Andrea Aristizábal Fúquene

Descripción: En esta investigación se presentan aspectos relacionados con la construcción y reconstrucción de las competencias interpretar, argumentar y proponer; siguiendo críticamente lo planteado por los estándares de competencias 2004. La investigación se realizó en el Colegio Gimnasio El Cedro y las temáticas objeto de trabajo fueron los conceptos de calor y temperatura; para lo cual se diseñaron y validaron tres instrumentos (cuestionario de concepciones alternativas, metarrelatos y la nave desconocimiento) para identificar el proceso de reconstrucción y construcción de las competencias mencionadas. Dicho trabajo se realizó a partir de un estudio histórico-epistemológico de los científicos que formularon las teorías; además, un análisis acerca de los aspectos sociales, políticos y culturales, en el momento de la publicación en revistas especializadas de la teoría de los conceptos en cuestión.

Contenidos: Esta investigación está constituida por unos antecedentes investigativos, lo epistemológico, el examen histórico de los conceptos de calor y temperatura; de la didáctica de las ciencias como disciplina conceptual y metodológicamente fundamentada, se establece una mirada sobre las competencias cognoscitivas en general y se delimita lo que se entiende por competencias interpretar, argumentar y proponer, se hace un aná- 
lisis del problema de los estándares y se instaura la propuesta en cuanto a la didáctica de las ciencias experimentales, particularizando los conceptos de calor y temperatura.

Metodología: Se construyó una versión pedagógica y didáctica para la enseñanza de los conceptos de calor y temperatura, en el marco de las competencias interpretar argumentar y proponer, siguiendo críticamente lo planteado por los estándares de competencias 2004. Se aplicó un cuestionario de concepciones alternativas para identificar las competencias cognoscitivas con las cuales ingresaron las estudiantes al proceso didáctico; después se organizaron grupos de trabajo y se les entregó el material de los artículos originales para interpretación, discusión y análisis. Además, se explicó la dinámica del trabajo, es decir, cómo se realiza la construcción de un metarrelato y cómo se resolvía la misión de la nave del conocimiento.

Se realizaron exposiciones en dónde se leyó el metarrelato, se explicaron los fundamentos de la teoría y se realizó un análisis del contexto social, político y económico del momento de la publicación de la teoría; después de abordar todos los metarrelatos se inició con la resolución de la nave del conocimiento en la que las estudiantes sustentaron de forma oral su solución y dejaron un documento escrito de dicha misión.

Conclusiones: La implementación de una propuesta didáctica de los conceptos de calor y temperatura permitió que las estudiantes reconstruyeran las competencias in- terpretar, argumentar y proponer con las que ingresaron al proceso, por cuanto elaboraron un modelo explicativo a partir de una narrativa oral y escrita diferente, de acuerdo con lo admitido por la comunidad de especialistas.

Seguir críticamente lo planteado por los estándares de competencias, desde una versión constructivista, contribuyó a cambiar la imagen de ciencia planteada por dichos estándares por cuanto no se partió de la convicción de que la construcción del conocimiento parte de la observación y de la interacción con el entorno, sino de elaboraciones teóricas que se han construido con el transcurso del tiempo y se han venido transformando.

Los instrumentos de recolección de información se validaron con la consulta de expertos según sus recomendaciones y aportaron como estrategia didáctica en el proceso de reconstrucción-construcción de las competencias interpretar, argumentar y proponer de las estudiantes. El proceso de transposición didáctica que elaboraron las estudiantes contribuyó a que las competencias IAP, entrarán en la dinámica de reconstrucción-construcción, por cuanto intervinieron, regularon y transformaron desde sus propios modelos explicativos la temática objeto de trabajo.

Los instrumentos (metarrelatos y la nave del conocimiento) respondieron de manera exitosa al proceso de reconstrucción-construcción de las competencias IAP y de las dimensiones que ellas implican en lo cognoscitivo, actuacional y motivacional, por cuanto se sintieron inmersas en dicho proceso. Las temáticas planteadas por los estándares de competencias que involucran los conceptos 
de calor y temperatura quedaron establecidas a partir de un estudio histórico-epistemológico riguroso, acorde con el nivel, para una mejor comprensión de los fenómenos.

\section{La formación inicial de profesores de química y una experiencia didáctica innovadora}

Liz Mayoly Muñoz Albarracín

Descripción: Se propone desarrollar una investigación en el campo de la formación inicial de profesores de química en la Universidad Pedagógica Nacional. Se parte del diseño y aplicación de los instrumentos que permitieron la identificación y caracterización de las concepciones epistemológicas, didácticas y pedagógicas con las que iniciaron estos estudiantes, y como consecuencia, se diseñó y desarrolló una estrategia desde la nueva didáctica de las ciencias, con miras a transformar estas concepciones; los resultados obtenidos de la contrastación de los instrumentos iniciales y finales se constituyeron durante el proceso en los indicadores de cambio y permitieron identificar la tendencia del grupo en cuanto a la posición empiropositivista y constructivista.

Contenido: El documento está conformado por la introducción, la justificación y la importancia, apartados desde donde se explica la pertinencia de realizar una investigación en el campo de la formación inicial de profesores de química en la Universidad Pedagógica Nacional. El marco teórico que comprende los antecedentes, los fundamentos epistemológicos, didácticos y pedagógicos, así como las temáticas que desde la nueva didác- tica de las ciencias aportan al desarrollo de la investigación en la formación inicial de profesores de ciencias en general y desde la química en particular.

Marco metodológico: Se formula como problema el de identificar, caracterizar y transformar las concepciones acerca de la epistemología, la didáctica y la pedagogía de los estudiantes que inician su formación como licenciados en química en la Universidad Pedagógica Nacional y la realización de una experiencia didáctica innovadora, para lo cual se hizo necesario diseñar los instrumentos para la recolección de la información, y se formularon las estrategias y los criterios de análisis. En lo epistemológico, se hará una propuesta acerca de la química como ciencia; en lo didáctico, se disertará sobre el campo de la formación inicial y continua de profesores y el diseño de una experiencia innovadora, y en lo pedagógico se abordará lo que tiene que ver con la educación, lo educativo y la educabilidad de las ciencias.

Conclusiones: En relación con las concepciones epistemológicas, didácticas y pedagógicas iniciales, estas fueron identificadas y caracterizadas para ser contrastadas con las nuevas elaboraciones que hicieron los estudiantes al final del proceso, lo que sirvió para emitir un juicio en torno a los cambios producidos en sus estructuras conceptuales, metodológicas, actitudinales y axiológicas (ECMAAs).

Los resultados mostraron que en cuanto a las concepciones epistemológicas, aunque existe una tendencia del grupo en general hacia una posición constructivista, 
existe la duda de qué tanto se alejan del empiropositivismo. En relación con las concepciones acerca del proceso enseñanza-aprendizaje, existen discordancias entre las dos, ya que en cuanto al aprendizaje la tendencia es constructivista y en la enseñanza no se estableció ninguna posición, y finalmente en lo pedagógico se da el caso de que se apoyan las dos posiciones, tanto la empiropositivista como la constructivista. El proceso de contrastación de las concepciones iniciales con las finales se estableció como criterio fundamental para identificar los cambios producidos en los estudiantes durante el trabajo realizado.

En cuanto al proceso de transformaciones de las concepciones de los estudiantes es necesario que la formulación de las estrategias didácticas sean coherentes con los objetivos de la investigación, ya que los procesos de cambio son complejos y pueden darse en la medida en que exista un seguimiento cuidadoso del proceso.

\section{La transposición didáctica del modelo de la estructura química del ADN. Un análisis en textos de enseñanza universitaria}

Angélica María García Torres

Descripción: Esta tesis de grado orientó la investigación de la transposición didáctica de la propuesta del modelo de la estructura química del ADN en relación con lo mostrado en los textos de enseñanza universitaria de mayor uso por parte de los docentes de la Escuela de Ciencias Químicas de la Universidad Pedagógica y Tecnológica de Colombia, UPTC, bajo el espacio académico denominado bioquímica.

Contenido: Esta investigación contiene un análisis sobre la transposición didáctica del modelo de la estructura química del ADN desde la confiabilidad de los textos de enseñanza universitaria con unos objetivos orientados a lo didáctico, lo pedagógico y lo epistemológico; un marco conceptual que contempla unos antecedentes relativos al campo de investigación de la transposición didáctica, un análisis histórico-epistemológico de la elucidación del modelo de la estructura química del ADN, unos fundamentos epistemológicos, pedagógicos y didácticos que fueron base del desarrollo de la presente investigación, una metodología fundamentada en los supuestos conceptuales y metodológicos del Grupo de Investigación, Representaciones y Conceptos Científicos, IREC, de la Universidad Pedagógica Nacional, unos resultados que pretendieron reconocer el modelo didactizado que comúnmente se expone en los textos universitarios frente a la temática objeto de trabajo. Finaliza la investigación con unas conclusiones en torno al trabajo en transposición didáctica dentro de la praxis formativa e investigativa del docente.

Metodología: El diseño y la aplicación de redes sistémicas particulares para cada uno de los textos seleccionados teniendo en cuenta tres dimensiones: la ontológica, la epistemológica y la de los distractores con respecto a la temática de la estructura química del $A D N$, para posteriormente desarrollar una red sistémica general 
entre el modelo científico, presentado por una comunidad especializada, y su correspondiente modelo didactizado evidenciado en los diferentes textos universitarios. Lo anterior con el fin de estipular bajo qué criterios de relación o diferenciación se desarrolla el proceso transpositivo en torno al tema objeto de investigación; sumado a ello, se presenta el uso de un instrumento en torno a las implicaciones histórico-epistemológicas y didáctico-pedagógicas que exponen los textos.

Conclusiones: Todo trabajo en transposición didáctica de un concepto o modelo científico implica una propuesta didáctica; el análisis de este instrumento es una posible pauta para la investigación, comprensión y control de los procesos de enseñanza-prendizaje del concepto o modelo científico.

Los textos didácticos seleccionados permitieron entrever una imagen de ciencia, en general, y de la propuesta del modelo de la estructura química del ADN, en particular, de manera lineal, operativa y conceptual; porque se orienta a estudiantes de formación profesional avanzada con respecto a la ciencia. Presentan como propuesta de enseñanza del tipo tradicional en la que no existen amplias posibilidades para la formación, tanto de científicos como de actitudes hacia la ciencia. Caen en el reduccionismo al suponer que la población a la que le interesa la temática relacionada con la genética, en general, y la estructura química del ADN, en particular, tiene la suficiente formación académica para la interpretación y comprensión de ella.
Existen diferentes criterios, tanto de relación como de distanciamiento, entre el modelo científico de la estructura química del ADN y su correspondiente modelo didactizado presente en los textos de enseñanza de la bioquímica a nivel universitario. Estos criterios pueden ser abordados desde las dimensiones ontológica y epistemológica de la temática que fue objeto de estudio.

El análisis de los conceptos o modelos científicos requiere de la relación y distinción entre los diferentes aspectos que lo componen. Su estructura puede ser interpretada en términos de su utilidad y predictibilidad bajo los referentes del concepto o modelo ya sea como un objeto de conocimiento (qué es y cómo se explica) o como una herramienta conceptual (qué permite explicar).

La transposición didáctica de un concepto o modelo científico, por efectos comunicativos, puede presentar dificultades para su interpretación. Por ello, el análisis de la transposición didáctica del modelo de la estructura química del ADN en los textos de enseñanza seleccionados puede demostrar posibles distorsiones, ambigüedades $\mathrm{y} / \mathrm{u}$ omisiones del modelo.

La transposición didáctica del modelo de la estructura química del ADN en los textos de enseñanza seleccionados no da la relevancia que les pertenece a la historia y filosofía de las ciencias, así como a la construcción metodológica, actitudinal y axiológica de quien decide tomar al texto como herramienta para su aprendiza- 
je. Además, no tiene en cuenta las ideas alternativas de los estudiantes. Tampoco dan relevancia a las interacciones CTS o a los efectos de la construcción y desarrollo del conocimiento científico. El estudio y el análisis de la confiabilidad de los textos didácticos indicaron que para los textos seleccionados se trata de una orientación empiro-positivista, acumulativa y lineal, que deja de lado los aspectos históricoepistemológicos, metodológicos y contextuales implicados en la construcción del conocimiento científico.

La presente investigación pretendió que, más allá de encontrar las falencias de los textos didácticos seleccionados, adquiere mayor relevancia determinar cuál es modelo didactizado que éstos conforman, su relación y diferenciación con el correspondiente modelo; para determinar dentro de nuestra praxis formativa e investigativa con docentes cuál ha de ser el papel del texto de enseñanza en de los procesos de enseñanza-aprendizaje. Se concluye que en la praxis académica del docente se hace necesaria la preocupación, necesidad y pertinencia del trabajo en transposición didáctica y de la confiabilidad de los textos.

\section{EnCUENTRo Nacional de Investigadores en Enseñanza de LAs Ciencias}

Invita

Abrapec, Asociación brasilera de pesquisadores en enseñanza de las ciencias

Bauru, Estado de San Pablo, Brasil

28 de noviembre al 03 de diciembre de 2005.

abrapec@fc.unesp.br 